\title{
A COMPREHENSIVE STUDY ON THEORETICAL AND EXPERIMENTAL EFFECTS OF NICOTINIC ACID AND PICOLINIC ACID ON THE STRUCTURE AND STABILITY OF HUMAN SERUM ALBUMIN
}

\author{
K. G. Chegini, ${ }^{a}$ S. M. Sadati, ${ }^{\text {b }}$ A. Rahbarimehr, ${ }^{b}$ \\ P. Yaghmaei, ${ }^{\text {A }}$ A. Farasat, ${ }^{\text {c }}$ and N. Gheibi ${ }^{a^{*}}$
}

UDC 543.42:547.962.3

The interaction of nicotinic acid (Nic) and picolinic acid (Pic), as two pyridine carboxylic acids, with human serum albumin (HSA) as a major transport protein in the blood was investigated using UV-Vis, fluorimetry, circular dichroism (CD), and molecular docking studies. The melting point $\left(T_{m}\right)$ and $\triangle G^{0}{ }_{(298 \mathrm{~K})}$ of HSA, as two thermodynamic parameters, were obtained from thermal denaturation of HSA with and without the presence of Nic and Pic. $T_{m}$ values of 332.5, 336.4 , and $333.9 \mathrm{~K}$, and $\Delta G^{0}{ }_{(298 \mathrm{~K})}$ of 97.4, 99.9, and $118.9 \mathrm{~kJ} / \mathrm{mol}$ were recorded for HSA alone and following incubation with Nic and Pic, respectively. In chemical denaturation experiments utilizing guanidine hydrochloride (GuHCl), value of $\Delta G^{0}{ }_{H 2 O}$ of $12.5,16$, and $15.3 \mathrm{~kJ} / \mathrm{mol}$, [Ligand] ${ }_{1 / 2}$ of 2.2, 2.4, and $2.3 \mathrm{M}$, and $\mathrm{m}$ of 5.6, 6.6, and $6.6 \mathrm{~kJ} /(\mathrm{mol} \times \mathrm{M})$ were recorded, respectively. The results of CD, UV-Vis spectroscopy, and molecular dynamics (MD) simulations showed that the binding of Nic and Pic to HSA induced conformational changes in HSA. Furthermore, the study of molecular docking indicated that the binding affinity of the Nic and Pic to site I (subdomain IIA) is greater than that of site II (subdomain IIIA) of HSA. These results provide valuable insights into the binding mechanisms of Nic and Pic to a plasma protein that is known to play an important role in the delivery of drugs to target organs.

Keywords: human serum albumin, stability, molecular docking, molecular dynamics.

Introduction. Pyridine carboxylic acid and its derivatives constitute several natural and synthetic products with special interest for medicinal chemists due to their physiological properties [1]. Currently, they have applications in biodegradable polymers and pharmaceuticals and as raw material for food additives. Nicotinic acid (3-pyridine carboxylic acid) and picolinic acid (2-pyridine carboxylic acid) are important chemicals used in the food and drug industries [1].

Nicotinic acid (also known as niacin, or vitamin B3) is a water-soluble vitamin with good biological activities and versatile bonding modes (Fig. 1a). It plays a nutritional role as a vitamin, a deficiency of which results in pellagra. This vitamin is involved in a wide range of biological processes, including production of energy, synthesis of fatty acids and steroids, signal transduction, regulation of gene expression, and maintenance of genomic integrity. It serves as a precursor to various forms of coenzyme nicotinamide adenine dinucleotide and a broad spectrum of lipid-based drugs applied to lower the cholesterol level [2].

Nicotinic acid derivatives are routinely used as antimicrobial, fungicidal, agricultural and industrial chemicals and have mostly been documented for their wide variety of pharmacological activities such as antimicrobial, analgesic, anti-inflammatory, anti-HIV, and antitubercular [3-7]. Picolinic acid is a white crystalline solid (Fig. 1a) with several applications in the pharmaceutical and chemical industries. It works as a chelating agent of molybdenum, zinc, chromium, manganese, and iron copper elements in the human body. Picolinic acid is a precursor for the production of pharmaceuticals such as local anesthetics and nutritional supplements such as metal salts [1]. Various biological media including, human milk, blood serum, cerebrospinal fluid (CSF), cell culture supernatants, intestinal homogenates, and pancreatic juice contain high levels of picolinic acid [8]. It is also used for quantitative detection of calcium. Picolinic acid is biosynthezed from tryptophan in the kidneys and liver, then stored and secreted in the pancreas and the intestine, respectively [9].

As the functional modules of the cell and the most abundant macromolecules in living systems, investigation of proteins is of extreme importance in life sciences, biochemistry and clinical medicine. Human serum albumin (HSA) is the

${ }^{\mathrm{a} C}$ Cellular and Molecular Research Center, Qazvin University of Medical Sciences, Qazvin, Iran; email: ngheibi@

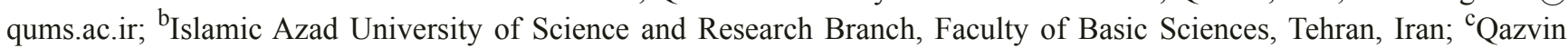
University of Medical Sciences, Department of Biotechnology, Qazvin, Iran. Abstract of article is published in Zhurnal Prikladnoi Spektroskopii, Vol. 86, No. 4, p. 666, July-August, 2019. 